\title{
Patterns of Transfer of Adaptation Among Body Segments ${ }^{*}$
}

\author{
${ }^{1}$ Motor Control Laboratory \\ ESPE Department \\ Arizona State University \\ P.O. Box 870404 \\ Tempe, AZ 85287-0404
}

\author{
${ }^{2}$ Life Sciences Research Laboratories \\ NASA/Johnson Space Center \\ Houston, TX 77058
}

${ }^{3}$ Currently at: University of Minnesota Dept of.Neuroscience
and Brain Sciences Center, $11 \mathrm{~B}$
Veterans Affairs Medical Center
Minneapolis, MN 55417

Mailing address for proofs: George E. Stelmach

Motor Control Laboratory

P.O. Box 870404

Tempe, AZ 85287-0404

email: stelmach@asu.edu

fax: 602/965.8108

phone: $602 / 965.9847$

*Portions of these data were presented in poster format at the 1998 Society for

Neuroscience annual meeting, Los Angeles, CA and the 1999 Society for the Neural

Control of Movement annual meeting, Kauai, HI. 


\begin{abstract}
Two experiments were conducted in order to determine the patterns of transfer of visuomotor adaptation between arm and head pointing. An altered gain of display of pointing movements was used to induce a conflict between visual and somatosensory representations. Two subject groups participated in Experiment One: group 1 adapted shoulder pointing movements, and group 2 adapted wrist pointing movements to a 0.5 gain of display. Following the adaptation regimen, subjects performed a transfer test in which the shoulder group performed wrist movements and the wrist group performed shoulder movements. The results demonstrated that both groups displayed typical adaptation curves, initially undershooting the target followed by a return to baseline performance. Transfer tests revealed that both groups had high transfer of the acquired adaptation to the other joint. Experiment Two followed a similar design except that group 1 adapted head pointing movements and group 2 adapted arm pointing movements. The arm adaptation had high transfer to head pointing while the head adaptation had very little transfer to arm pointing. These results imply that, while the arm segments may share a common target representation for goal-directed actions, individual but functionally dependent target representations may exist for the control of head and arm movements.
\end{abstract}


Introduction

Subjects can readily adapt both goal-directed eye and arm actions in response to visuomotor conflict, such as occurs with prismatic lenses (cf. Harris, 1965; Held, 1961, 1965), the double-step target displacement paradigm (cf. de Graaf et al., 1995; Abrams et al., 1992), and rotated visual feedback (cf. Cunningham \& Welch, 1994; Kagerer et al., 1997), resulting in an updated mapping between visual space and motor space. The patterns of transfer of this adaptation between body segments have been studied in an effort to determine whether multiple segments share a common visuomotor mapping. For example, de Graaf et al. (1995) demonstrated that saccadic adaptation transferred to arm pointing movements, and proposed as a potential mechanism the idea that the eyes and the arm represent target locations in a common visuomotor map, potentially centered on the eyes. If all body segments were to share this same mapping, then adaptation of any one segment should transfer to the others.

An alternative interpretation to that provided previously, however, is that multiple mappings exist, and that they are arranged in a hierarchical manner. This is supported by the findings that, if subjects point with one arm while wearing displacing prisms (with the head fixed), transfer of adaptive modifications does not occur to the other arm (Prablanc et al. $1975 \mathrm{a}, \mathrm{b})$. However, if subjects point by moving the head to targets or if the head is unrestrained during arm pointing, then transfer occurs to both arms (Wallace 1978; Jeannerod 1988; Redding and Wallace 1988). These data suggest that the head-centered target representation may be preferential to that of the arm, potentially providing a stable reference for the visual and vestibular systems (Pozzo et al. 1990; Prablanc et al. 1975a,b; Jeannerod 1988). Further support of this idea is provided by Bloomberg et al. (2000). In 
their experiment, the gain of the vestibulo-ocular reflex response was adaptively reduced by having subjects wear 0.5 minifying lenses and perform head movements with the arms restrained and out of view for 30 minutes. Transfer to the manual aiming system was assessed by having subjects point to a remembered target following passive, whole body rotation in the dark without the lenses. The ensuing reduced gain of the pointing response suggests that adaptation is transferred in a hierarchical fashion. Similarly, the combined work of Hay and Brouchon (1972) and Putterman et al. (1969) suggests that adaptation of arm pointing movements while wearing displacing prisms transfers to the wrist, but wrist adaptation does not transfer to the arm. These studies suggest that multiple mappings exist, and that adaptive modifications occurring to a map for a particular body segment will transfer to mappings of the segments that are located distally or below it. Additionally, top-down transfer suggests the existence of two mappings that are functionally dependent. That is, if a more proximal segment is adapted it will influence distal mappings. When the distal segments are adapted there is no change to the proximal mappings.

The previous studies addressing transfer of adaptation have either invoked adaptation by altering the gain of saccades or by having subjects wear some type of lens over the eyes (displacing prisms, minifying or magnifying lenses). In the case of minifying or magnifying lenses, the vestibulo-ocular reflex (VOR) will be updated in addition to adaptive modifications in head pointing. Furthermore, prism lenses introduce an oculomotor bias when the subject attempts to look straight ahead. This likely changes the internal representation of the straight-ahead position. It is unclear whether the hierarchical pattern of transfer of adaptation would be observed for adaptation in 
response to strictly a modified display of feedback (i.e., gain change or rotated feedback displayed to subjects). This would result in adapted pointing responses without changing gaze angle, or inducing oculomotor bias. Furthermore, it remains unknown whether head adaptation follows the top-down transfer patterns when it occurs without the oculomotor bias introduced by prism lenses. Clearly, the hierarchy hypothesis predicts that it would. Thus, the purposes of the two experiments reported were twofold. One was to determine whether transfer of adaptation follows a top-down pattern when the adaptive stimulus is a change in gain of display of pointing movements. The other purpose was to determine whether head adaptation follows the top-down transfer pattern when it occurs without an accompanying oculomotor bias.

\section{Experiment One}

It is unclear whether one or more target representations are used for movements of the multiple arm segments. Previous work suggests that multiple mappings exist in a hierarchical arrangement. That is, adaptation of pointing movements while subjects were wearing prisms transfers from the arm to the wrist but not the wrist to the arm (Putterman et al., 1969; Hay and Brouchon, 1972). The purpose of Experiment One was to determine whether similar patterns of transfer are seen when subjects adapt pointing movements in response to a change in gain of display. Subjects moved a stylus over a digitizing tablet. A cover over their arm prevented them from directly viewing their movements. Instead, they viewed a pen trace moving on a monitor directly in front of them (Figure 1). Thus, there were no resulting adaptive changes in eye movements or resting angle of gaze. High transfer of this adaptation between the shoulder and the wrist would support the hypothesis that both joints share a common target representation. In 
contrast, if there is little or no transfer of adaptation between joints, the results would imply that each has access to its own unique visuomotor mapping. A hierarchical arrangement of multiple representations would be supported if shoulder adaptation transfers to wrist pointing without wrist adaptation transferring to shoulder pointing movements, as observed in the case of prismatic adaptation. This would imply that the shoulder and the wrist each have their own mapping, but that of the shoulder is relied upon predominantly to that of the wrist.

Methods

Subjects. Two groups of sixteen subjects were recruited from the Arizona State University campus to participate. Group 1 subjects ( 4 males, 12 females) were 22.2 $(\mathrm{sd}=2.5)$ years old; group 2 subjects ( 6 males, 10 females $)$ were $23.0(\mathrm{sd}=3.9)$ years old. After hearing an explanation of the experiment, the subjects provided written informed consent in accordance with the ASU institutional review board human subjects' policies. Subjects were compensated with extra credit for an undergraduate motor learning course for their participation, which took an average of one hour.

Procedure. Subjects were seated in front of a computer monitor, with their arm resting on a digitizing tablet as shown in Figure 1. The shoulder was flexed to $90^{\circ}$ for the starting posture, and the table height was adjusted to support the arm in this posture parallel to the floor. The head was fixed to a support using a molded head restraint and a headband for the duration of the experiment. Subjects grasped the stylus in a whole hand grasp with their arm under a cover to prevent vision of the moving hand. The subjects performed aiming movements to various targets on a CalComp (GTCO CalComp Inc., Scottsdale, AZ)-digitizer. Custom software was used to present the imperative stimulus, 
the targets, and the pen trace in real time, and to collect the $\mathrm{x}$ and $\mathrm{y}$ pen tip coordinates at $100 \mathrm{~Hz}$ during the course of the movements. Targets and the pen trajectory were displayed on a $30 \mathrm{~cm}$ by $40 \mathrm{~cm}$ monitor, placed at eye level $60 \mathrm{~cm}$ back from the testing chair. The subjects were instructed to perform the movements as rapidly and as accurately as possible upon an auditory go signal. Although latency of response was not stressed, anticipation and no response trials were omitted by having subjects repeat trials in which they did not achieve a reaction time (RT) between $100 \mathrm{~ms}$ and $1000 \mathrm{~ms}$. All other trials were retained, regardless of whether or not the target was achieved.

Group 1 (shoulder adaptation, SA) performed either shoulder horizontal flexion or extension to move from the home position to the target, while group 2 (wrist adaptation, WA) performed either wrist flexion or extension. Elbow and shoulder motions were constrained for the WA group. Similarly, the SA group's movements were limited to horizontal flexion and extension only by placing one brace on the elbow and another on the wrist. Both groups performed a pre test with the gain of display at 1.0, an adaptation block with the gain at 0.5 , and a transfer post test in which the groups switched effectors, with the gain remaining at 0.5 (see Table 1 for an overview of trial presentation). The 0.5 gain of display requires subjects to travel twice the distance on the tablet compared to the distance displayed on the monitor. For all blocks, subjects performed half of the trials in one movement direction first (flexion or extension), and moved in the opposite direction for the remainder of the trials. After achieving the target, subjects returned to the start position. The presentation of flexion or extension trials first was counterbalanced across subjects. 
Subjects performed 15 trials for each of the two accommodation blocks (block 1a consisting of flexion movements and $1 \mathrm{~b}$ consisting of extension movements), in order to familiarize themselves with the digitizer tablet and pen. Following this, both groups performed six adaptation blocks (blocks A1a \& b, A2a \& b, A3a \& b) in which the gain of display was reduced with respect to the actual movements performed by a factor of 0.5. The movement amplitude changed with every other block, and movement direction changed with each block, as shown in Table 1. Varying movement amplitudes were used to help lead to generalization and consolidation of the adaptive state. A total of 90 adaptation trials were performed. The number of trials performed within each adaptation block was reduced with successive blocks because fewer trials were required before performance stabilized. Following this adaptation period, both subject groups performed two blocks of transfer trials (block 3a consisting of flexion movements \& $3 \mathrm{~b}$ consisting of extension movements) in which the gain of display remained at 0.5 . The WA group performed shoulder movements for the transfer blocks and the SA group performed wrist movements. The movement amplitude was also changed when going from the adaptation block to the transfer test (Table 1). This was done because the maximum distance that could be covered with the wrist joint was much smaller than that with the shoulder joint. Furthermore, it was predicted that this would not reduce the chances of transfer occurring due to the variety of movement amplitudes that subjects experienced during the adaptation blocks.

Data Analysis. The pen position data were subjected to a residual analysis in order to determine the appropriate cutoff frequency for data filtering (Winter 1990); the resulting value ased was $7 \mathrm{~Hz}$. The trajectory over time was computed from the radius 
vectors, which are the vectors from the origin to the spatial location of the pen tip at each point in time. The trajectory is computed as the length of these vectors over time (the square root of the sum of the squared $\mathrm{x}$ and $\mathrm{y}$ coordinate data at each point in time). The tangential speed and acceleration profiles were then found by successive differentiation of this trajectory. The optimal algorithm of Teasdale et al. (1993) was used to determine movement onset from the speed profiles. The algorithm works as follows: Locate the sample at which the speed time series first exceeds $10 \%$ of its maximum value (Vmax); working back from this point stop at the first sample (call it S) less than or equal to $(V \max / 10)-(V \max / 100)$; find the standard deviation of the series between sample 1 and sample S (call this sd); working back from $S$ stop at the first sample less than or equal to S-sd; this is the onset sample. As sampling was terminated when subjects remained stationary for $300 \mathrm{~ms}$, the same algorithm was used in reverse to determine movement offset.

Subjects typically perform movement corrections during adaptation experiments, evident as corrective submovements in the velocity profile (cf. Meyer et al. 1988; Kagerer et al. 1997), especially towards the beginning of the adaptation blocks when they are learning the task and adapting to the change in the gain of display. Since the focus of interest was more in how the subjects preplanned the movements and less in the on-line corrections that they made, we decomposed the movement into its primary and secondary submovements. The primary submovement is thought to be mostly under ballistic control whereas the secondary submovement for the most part reflects a feedback-based correction (Meyer et al. 1988). The existence of secondary submovements was determined using an algorithm that searches for a positive acceleration value following a 
period of deceleration, or a change in the sign of the velocity, signifying a change in movement direction (Carson et al. 1992; Elliott et al. 1991; Walker et al. 1997; SeidlerDobrin and Stelmach 1998). The end of the primary submovement was also considered the beginning of the secondary submovement. While it is acknowledged that multiple corrective submovements may occur, they were considered as one corrective phase for the purposes of this analysis.

Using these methods of submovement decomposition allowed us to portion the movement into primary and secondary components. This enabled us to compute the distance covered in the primary submovement, reflective predominantly of motor programming errors rather than errors in any feedback-based corrections. These distances were normalized as a percentage of the target distance for interpretation purposes. For example, the primary submovement should cover approximately $50 \%$ of the total distance for the initial trials of the adaptation block.

A within subjects MANOVA (group $\times$ block $\times$ trial) with repeated measures on trial and block was used to determine how performance varied across each block. Since the number of trials varied across blocks, only the first 10 trials from each block were entered into the analyses. The Huynh-Feldt epsilon (Huynh and Feldt 1970) was evaluated to determine whether the repeated measures data met the assumption of sphericity $(\Sigma>.75)$. In cases where sphericity was met, the univariate tests were used to maintain power. Otherwise, the repeated measures were treated as multivariate. Note that the significance of the $\mathrm{F}$ ratio is assessed using different degrees of freedom depending on whether the univariate or the multivariate tests are used. The observed power was computed for all effects, as was $\omega^{2}$, an estimate of the total population 
variance that is explained by the variation due to the treatment (Keppell, 1991). Its value does not depend on sample size or power of the experiment. Its values can range between 0.0 and 1.0, with negative values a possibility when the associated $F$ value is less than 1.0. Cohen suggests that a small effect is comparable to an $\omega^{2}$ of .01 , a medium effect is .06, and a large effect is .15 or greater (Cohen, 1977). These standards were employed in our assessment of treatment effect sizes.

Trend analyses were performed over the trials in the adaptation block and the trials in the transfer block. Performance during adaptation was expected to show significant linear and quadratic trends. If transfer of adaptation is high, then there would be no need for adaptive changes during the transfer blocks. If this is the case, then there would not be significant linear and/or quadratic trends describing the data during the transfer blocks. Follow-up comparisons were at an adjusted alpha rate of .01. Whether the primary submovement distance was significantly greater than $50 \%$ of the target distance at the beginning of the transfer blocks was assessed using confidence intervals. Results

The observed power for all significant reported effects ranged from .80 to .99 . There was no significant effect of initial movement direction (flexion vs. extension trials first), so all results were pooled across this factor. The overall group $\times$ block $\times$ trial interaction was not significant for primary submovement distance $(\mathrm{F}<1.0)$; however the block $\times$ trial interaction was $\left(\mathrm{F}_{81,2430}=2.71, \mathrm{p}<.001, \omega^{2}=.27\right.$, large effect size). Therefore, follow-up tests were performed within each block. There were no significant interactions or main effects $(\mathrm{F}<1.0)$ for the accommodation trials. Both groups covered 
approximately $90 \%$ of the target distance with the primary submovement by the end of the pre test blocks.

Figure 2 plots sample trials from early and late in adaptation and the initial transfer test trials for both groups. Qualitative changes can be seen in the adjustments made to the trajectory when comparing early (upper row) with late (middle row) adaptation trials. Figure 3 plots the primary submovement data for both groups during the adaptation blocks. A total of six trend analyses were preformed for the adaptation data. There were no group differences in trend during adaptation. There were significant linear and quadratic trends across trials in adaptation blocks A1a and A2a (Linear: block A1a $F_{1,30}=136.5, p<.001, \omega^{2}=.68$, large effect size, block A2a $F_{1,30}=12.5, p<.01, \omega^{2}$ $=.15$, large effect size, Quadratic: block A1a $\mathrm{F}_{1,30}=103.2, \mathrm{p}<.001, \omega^{2}=.61$, large effect size, block $A 2 \mathrm{a} \mathrm{F}_{1,30}=35.5, \mathrm{p}<.01, \omega^{2}=.35$, large effect size), and linear trends in blocks A1b, A2b, and A3a (block A1b $\mathrm{F}_{1,30}=16.2, \mathrm{p}<.001, \omega^{2}=.19$, large effect size, block A2 $\mathrm{bF}_{1,30}=6.3, \mathrm{p}<.02, \omega^{2}=.08$, medium effect size, block A3a $\mathrm{F}_{1,30}=16.3, \mathrm{p}<$ $.001, \omega^{2}=.19$, large effect size). Note that there were no longer any trends over trials for the last adaptation block (A3b).

The sample trajectories from the first trial of transfer to the new effector are qualitatively similar to those presented for late in adaptation (Figure 2). Primary submovement distance during the transfer test is plotted in Figure 4. Again, there were no group differences in performance $\left(\mathrm{F}_{1,30}<1.0\right)$. Linear trends existed across trials for both of the transfer blocks (block $3 \mathrm{a} \mathrm{F}_{1,30}=10.0, \mathrm{p}<.01, \omega^{2}=.12$, medium effect size, block $3 b \mathrm{~F}_{1,30}=12.2, \mathrm{p}<.01, \omega^{2}=.15$, large effect size). The $95 \%$ confidence intervals (CI) for the primary submovement percentage on the first trial demonstrated that subjects 
covered substantially more than $50 \%$ of the target distance, with the CI for Group 1 (SA): $76.3-96.6 \%$ and that of Group 2 (WA): $73.8-92.5 \%$.

Discussion

Both subject groups (wrist and shoulder joint pointing) adapted to the 0.5 gain of display in a typical fashion, initially undershooting the target followed by a return to baseline performance. The transfer test demonstrated that both groups exhibited high transfer of the acquired adaptation to the new effector. These results suggest that the shoulder joint and the wrist joint may share a common target representation for goal directed actions. This would simplify the control process by limiting the number of sensorimotor transformations required. Moreover, a single target representation for movements of each arm segment may aid the coordination of proximal and distal arm segments, despite the differing contributions that they make to multijoint arm movements (Dounskaia et al. 1998; Seidler \& Stelmach, in press).

The patterns of transfer of adaptation obtained in this study differ from the results found when subjects adapt pointing movements while wearing wedge prisms (Hay \& Brouchon, 1972; Putterman et al., 1969). These authors found top-down transfer only. When subjects don prism lenses, an oculomotor bias is induced when they attempt to look straight ahead. Prisms may have a differential effect on proximal and distal arm segments, as the distal segments are controlled to a greater extent with visual guidance (refs to support this). Regardless of the mechanisms, it is clear that the mode of adaptation has a significant impact on the resulting transfer patterns. While our data do support the existence of a single target representation for goal-directed wrist and shoulder actions, we are unable to distinguish whether these movements are controlled from a 
shoulder-centered frame of reference, as has been previously suggested (Caminiti et al. 1991; Soechting and Flanders 1991; Darling et al. 1996; Darling and Hondzinski 1997; Lacquaniti et al., 1995), or a wrist- (Gordon et al., 1994) or eye-centered (de Graaf et al., 1995) reference frame.

Experiment Two

Evidence exists to suggest that sensorimotor transformations for arm pointing movements may begin with a retinotopic target representation, followed by transformation to a head-centered one, and finally to a shoulder-centered one for the execution of pointing movements (Gnadt et al. 1991; Soechting and Flanders 1991; Henriques et al. 1998; Graziano et al. 1997). It is unclear, however, whether these multiple representations function in a hierarchical fashion with respect to each other. It may be that the head-centered representation is relied upon predominantly with respect to the others because of the need to provide a stable reference frame for the visual and vestibular systems (Pozzo et al. 1990; Bloomberg et al. 1997).

The evidence used to support the top-down transfer hypothesis is data obtained using adaptation to either minifying lenses or displacing goggles (Prablanc et al. 1975a, b; Wallace 1978; Jeannerod 1988; Redding and Wallace 1988). However, prism lenses introduce an oculomotor bias when the subject attempts to look straight ahead, altering the internal representation of the straight-ahead position. It is unclear whether the topdown transfer pattern observed with prism adaptation would be obtained when subjects adapt to a change in gain of display. Additionally, it remains unknown whether adaptation of arm pointing movements would transfer to head pointing. Clearly, the hierarchy hypothesis (Pozzo et al. 1990; Prablanc et al. 1975a,b; Jeannerod 1988) 
predicts that it would not; the hypothesis that all body segments share a common retinotopic target representation (de Graaf et al., 1995), however, would predict transfer of adaptation from the arm to the head. Thus, the purpose of Experiment 2 was to examine transfer of adaptation to a change in gain of display between head and arm pointing movements.

Methods

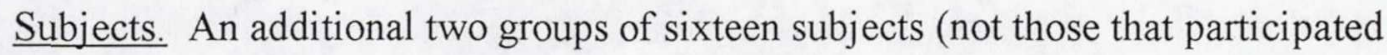
in Experiment 1) were recruited from the Arizona State University campus to participate. Group 1 subjects ( 7 males, 9 females) were $23.0(\mathrm{sd}=3.7)$ years old; group 2 subjects ( 9 males, 7 females) were 23.4 ( $\mathrm{sd}=2.8$ ) years old. After hearing an explanation of the experiment the subjects provided written informed consent in accordance with human subjects' policies. Subjects were again compensated with extra credit for an undergraduate motor learning course for their participation, which took an average of one hour.

Procedure. Subjects were seated in front of a computer monitor, with their arm resting on a digitizing tablet. Subjects were free to use any joints within the arm to achieve the task, as the results of Experiment 1 supported that only one coordinate system exists within the arm. The head was fixed to a support for the arm movement group only. The arm adaptation group (group 2, AA) grasped the stylus in a whole hand grasp with their hand under a cover to prevent vision of the moving hand. The head adaptation group (group 1, HA) wore a lightweight head band with the digitizing stylus attached, pointed towards the ceiling. The digitizing tablet was placed up side down on shelves above the subject's head. The chair height was raised until the stylus made contact with 
the tablet. Subjects in the HA group drew on the tablet by rotating their head towards the left and the right. A Wacom (Wacom Technology Corporation, Vancouver, WA) digitizer was used for Experiment 2. Custom software was used to present the imperative stimulus (an auditory go signal), the targets, and the pen trace in real time, and to collect the $\mathrm{x}$ and $\mathrm{y}$ pen tip coordinates at $206 \mathrm{~Hz}$ during the course of the movements. Targets were displayed on a $30 \mathrm{~cm}$ by $40 \mathrm{~cm}$ monitor, placed at eye level $60 \mathrm{~cm}$ back from the subjects.

The paradigm was similar to that used for Experiment 1, except that both groups received four initial accommodation blocks rather than two, two drawing with the arm and two drawing with the head (block 1a consisting of left-to-right movements \& $1 \mathrm{~b}$ consisting of right-to-left movements, and block 2a consisting of left-to-right movements $\& 2 \mathrm{~b}$ consisting of right-to-left movements). These initial tests were performed with the gain of display at 1.0. The six adaptation (blocks A1a \& b, A2a \& b, A3a \& b) and two transfer blocks (block 4a \& 4b) performed by both groups followed the same design as in Experiment 1. Table 2 presents an overview of trial presentations for Experiment 2.

Data Analysis. The data analysis procedures were identical to those in Experiment 1.

Results

The observed power for all significant effects ranged from .78 to .99 . The overall group $\times$ block $\times$ trial interaction for primary submovement distance was significant $\left(\mathrm{F}_{99}\right.$, $270=2.03, \mathrm{p}<.001, \omega^{2}=.03$, small effect size); therefore group $\times$ trial follow-up tests were conducted within each block. For both blocks of the first accommodation test (block 1a \& 1b) there was a significant group $\times$ trial interaction of the linear trend over 
trials (block 1a $\mathrm{F}_{1,30}=17.6, \mathrm{p}<.001, \omega^{2}=.21$, large effect size, block $1 \mathrm{~b} \mathrm{~F}_{1,30}=8.4, \mathrm{p}<$ $.01, \omega^{2}=.10$, medium effect size), reflecting that group 1 (HA) increased performance at a faster rate than group $2(\mathrm{AA})$. For both blocks of the second accommodation test, there was a group main effect (block $2 \mathrm{a} \mathrm{F}_{1,30}=6.8, \mathrm{p}=.01, \omega^{2}=.15$, large effect size, block $2 \mathrm{~b}$ $\mathrm{F}_{1,30}=6.7, \mathrm{p}=.01, \omega^{2}=.15$, large effect size), reflecting that group $2(\mathrm{AA})$ covered a greater percentage of the target distance with the primary submovement than group 1 (HA). Moreover, there was a significant linear trend over trials for both groups in each of the two blocks (block $2 \mathrm{a} \mathrm{F}_{1,30}=45.5,<.001, \omega^{2}=.41$, large effect size, block $2 \mathrm{~b} \mathrm{~F}_{1,30}=$ $14.4, \mathrm{p}<.01, \omega^{2}=.17$, large effect size)

Figure 5 plots individual trajectories for trials early and late in adaptation and upon transfer. As in Experiment One, the late adaptation trajectories do not exhibit the adjustments that are evident early in adaptation. Primary submovement performance across the six adaptation blocks is depicted in Figure 6. There were significant linear and quadratic trends across trials for the first two blocks (Linear: block A1a $F_{1,30}=30.7$, $\mathrm{p}<.001, \omega^{2}=.32$, large effect size, block $\mathrm{Alb} \mathrm{F}_{1,30}=12.8, \mathrm{p}<.01, \omega^{2}=.15$, large effect size, Quadratic: block A1a $\mathrm{F}_{1,30}=10.2, \mathrm{p}<.01, \omega^{2}=.13$, medium effect size, block A1b $\mathrm{F}_{1,30}=9.0, \mathrm{p}<.01, \omega^{2}=.11$, medium effect size). The only other effect observed during adaptation was a group main effect in block $\mathrm{A} 2 \mathrm{~b}\left(\mathrm{~F}_{1,30}=14.4, \mathrm{p}<.01, \omega^{2}=.17\right.$, large effect size).

Group 1 (HA) exhibited adjustments upon transfer to arm drawing similar to those seen early in the adaptation process, while group 2 (AA, transfer to head drawing) did not (Figure 7). There was a significant group $\times$ trial interaction for distance covered in the primary submovement for the first transfer test block (block $4 \mathrm{a} \mathrm{F}_{9,270}=4.5, \mathrm{p}<.001, \omega^{2}=$ 
.33 , large effect size) (Figure 8). Follow up tests revealed a group difference in the linear trend across trials in this block $\left(\mathrm{F}_{1,30}=25.6, \mathrm{p}<.001, \omega^{2}=.28\right.$, large effect size $)$, reflecting the changing performance of group 1 (HA) during this block while group 2 (AA) remained constant. The $95 \%$ confidence intervals for performance on the first trial of the transfer test were only slightly overlapping between the two groups, with the group 1 (HA) interval including $50 \%$ of the distance covered with the primary submovement distance: $43.5-62.9 \%$, while the interval for group 2 (AA) did not: 58.7 $-87.8 \%$. Although there were no significant group differences in the primary submovement distance for the last two adaptation blocks, one may argue that the HA group covered less distance and therefore the $50 \%$ transfer assessment is inappropriate. The average of the last three data points in block $\mathrm{A} 3 \mathrm{~b}$ is $85.1 \%$ for the HA group and $91.0 \%$ for the AA group. For the first transfer trial, the HA group covers $50.5 \%$ of the target distance drawing with the arm and the AA group covers $75.5 \%$ of the target distance drawing with the head. These values are $59 \%$ and $83 \%$ of the distance that was covered at the end of the adaptation block. Thus while it may be that some transfer occurs from the head to the arm, much greater transfer is observed from the arm to the head.

Discussion

As in Experiment One, both groups (head and arm pointing) adapted to the change in gain of display in a typical fashion, initially undershooting the targets followed by a rapid return of performance to baseline levels. In contrast to Experiment One, high transfer of adaptation occurred in only one group. Group 1, the head adaptation group, exhibited only little transfer of adaptation to arm pointing. This is evident in Figure 4 by 
the multiple corrections made in the trajectories for group 1 when switching to arm drawing. Group 2, however, demonstrated high transfer from arm pointing to head pointing. These results suggest that the head and the arm may not share a common coding of target location representation. This idea is supported by previous literature (Gnadt et al. 1991; Soechting and Flanders 1991; Jeannerod 1988).

Overall, there was a tendency (but not a significant group difference) for the HA group to cover a smaller percentage of the total distance with the initial ballistic submovement than the AA group did. This reflects the difficulty subjects experienced in pointing with the head. However, it is not likely that insufficient practice with head drawing contributed to the lack of transfer of adaptation from the head to the arm. The AA group had no practice in drawing with the head in the 0.5 gain exposure, and yet arm adaptation transferred to head pointing. Furthermore, the HA group demonstrated substantial evidence of adaptation, increasing the distance covered with the primary submovement well above $50 \%$ of the target distance. A Additionally, there were no group differences in performance during adaptation with the exception of one block.

Some investigators have demonstrated transfer of adaptation from the eye-head system to the arm (Bloomberg et al. 2000; Wallace 1978; Jeannerod 1988; Redding \& Wallace 1988). However, these investigations used either displacing prisms or minifying lenses to induce adaptive modifications. In contrast, this experiment changed the gain of display of movements on a computer monitor. To our knowledge, this is the first time that head pointing movements have been adapted to this type of stimulus. Using this task, we were able to modify only the visually-derived representation of the moving body segment, without simultaneously adapting the VOR as would have occurred if the 
subjects wore minifying or magnifying lenses (Miles and Lisberger 1981; Ito 1982;

Berthoz and Melvill Jones 1985). Additionally, this process does not result in the static oculomotor bias that prism lenses induce. It appears that oculomotor bias may induce a top-down adaptive scheme, while adaptation to a change in gain of display may cause a more generalized adaptive response with more opportunities for up and down transfer. This issue warrants further investigation. Furthermore, transfer of adaptation from the arm up to the head has not previously been examined. Presumably, if the adaptive stimulus were prism lenses the results would follow the patterns of top-down transfer only that have been observed within the arm and from the head to the arm (Wallace, 1978; Jeannerod, 1988; Redding \& Wallace, 1988; Hay \& Brouchon, 1972; Putterman et al., 1969).

General Discussion

While experiment one revealed symmetrical transfer of adaptation, experiment two did not. Adaptation transferred from the arm up to head pointing but not from the head to arm pointing in experiment two. The combined results of these two experiments suggest that the arm and the head do not share common coding of the target representation (Gnadt et al. 1991; Henriques et al. 1998; Soechting and Flanders 1991). Furthermore, the results of these experiments are consistent with a potential one-way direction of sensorimotor transformations that occur from the initial, retinotopic target representation to a head-centered one and finally to an arm-centered one for the execution of goal-directed actions. During adaptation of arm pointing movements to a change in gain of display, it is likely that the arm-centered target location mapping is updated. Thus, the algorithm used to transform target location from the head-centered to the arm- 
centered representation would also need to be updated. Even though the head is fixed during the production of these arm movements, it appears that the intermediate stage representation in a head-centered reference frame still occurs, resulting in an updating of this mapping as well. However, since the eyes are not fixed during the experiment, it is likely that the system does not update the retinotopic target representation but rather just modifies the transform from it to the head-centered one. This scheme is supported by the transfer that occurs from arm adaptation to head pointing movements, as observed in Experiment 2.

In the case of the head pointing adaptation group, there may only be a transformation from the retinotopic target representation to a head-centered one. During adaptation of head movements to a new gain, the head-centered target representation would be updated, and the transform from the retinotopic to the head-centered target representation. The idea that the arm-centered target representation is not updated is supported by the finding that head adaptation did not transfer to arm pointing.

With the head fixed during arm pointing, the question arises as to whether a headcentered target representation is actually invoked. It may be that this intermediate stage is skipped, with a direct transformation made from the retinotopic target representation to the arm-centered one. This scheme would be computationally simpler, because it would require only one transformation directly from the eye-centered to the arm-centered visuomotor map, bypassing the intermediate steps of transforming to and from a headcentered representation. We would argue, however, that this is not possible simply because arm adaptation transfers to the head while head adaptation does not transfer to the arm. If the head-centered target representation were not employed when the head is 
fixed with respect to the trunk, we would not see transfer from the arm to the head. Several authors have suggested that the sensorimotor transformation process could however be simplified by fixing the head to the trunk (Mergner et al. 1997; Darling and Miller 1995). We agree with this concept; in effect, fixing the head to the trunk would result in the transformation from one reference frame to another requiring only a simple displacement, without a rotation of axes. Interestingly, this head-on-trunk stabilization strategy appears to be employed by astronauts following spaceflight (Blooomberg et al. 1997b) and by vestibular patients (Pozzo et al. 1991).

The hierarchy hypothesis suggests that the target representation for head . movements dominates over that for the arm (Pozzo et al. 1990; Prablanc et al. 1975a, b; Jeannerod 1988), with any adaptive modification occurring in the head transferring to the arm. However, our data suggest that the target representation for the head does not appear to be preferential to that of the arm. It appears that the difference in the pattern of results in comparison to previous experiments arises due to the mode of adaptation employed, particularly whether subjects are viewing targets through lenses or are instead only viewing altered movement feedback (Bloomberg et al. 2000; Wallace 1978; Jeannerod 1988; Redding and Wallace 1988). These contrasting findings suggest that reference frames for target representations are likely highly flexible in nature and taskdependent.

Conclusions 
The results of Experiment 1 demonstrated high transfer of adaptation to a change in gain of display between shoulder and wrist pointing movements of the same arm. These data suggest that all arm segments share a common target representation for goaldirected actions. Experiment 2 revealed that arm adaptation transferred highly to head pointing but head adaptation transferred only little to arm pointing. These data suggest that individual but dependent target representations are used for goal-directed arm and head pointing movements, and that the target representation for head pointing is not used preferentially to that of the arm. 


\section{Acknowledgements}

This work was supported by NASA NGT9-21 and NIA AG14676. The authors wish to acknowledge the assistance of A. van Gemmert in OASIS programming for data acquisition in Experiment 2, and the valuable comments provided by James Bloedel, Edward Castaneda, and Philip Martin. 


\section{Table 1. Experiment One Trial Descriptions}

\begin{tabular}{|c|c|c|}
\hline Block Number & WA Group & SA Group \\
\hline Block la \& $1 \mathrm{~b}$. & $\begin{array}{l}15 \text { wrist flexion trials } \& 15 \\
\text { wrist extension trials, } 4.3 \\
\mathrm{~cm} \text { amplitude, gain }=1.0 .\end{array}$ & $\begin{array}{l}15 \text { shoulder flexion trials \& } \\
15 \text { shoulder extension trials, } \\
8.2 \mathrm{~cm} \text { amplitude, gain }=1.0 \text {. }\end{array}$ \\
\hline Ala \& A1b: & $\begin{array}{l}20 \text { wrist flexion trials } \& 20 \\
\text { wrist extension trials, } 3.8 \\
\mathrm{~cm} \text { amplitude, gain }=0.5 \text {. }\end{array}$ & $\begin{array}{l}20 \text { shoulder flexion trials \& } \\
20 \text { shoulder extension trials, } \\
5.4 \mathrm{~cm} \text { amplitude, gain }=0.5 \text {. }\end{array}$ \\
\hline $\mathrm{A} 2 \mathrm{a} \& \mathrm{~A} 2 \mathrm{~b}$ & $\begin{array}{l}15 \text { wrist flexion trials \& } 15 \\
\text { wrist extension trials, } 5.4 \\
\mathrm{~cm} \text { amplitude, gain }=0.5 \text {. }\end{array}$ & $\begin{array}{l}15 \text { shoulder flexion trials \& } \\
15 \text { shoulder extension trials, } \\
10.8 \mathrm{~cm} \text { amplitude gain }=0.5 \text {. }\end{array}$ \\
\hline A3a \& A3b: & $\begin{array}{l}10 \text { wrist flexion trials \& } 10 \\
\text { wrist extension trials, } 7.0 \\
\mathrm{~cm} \text { amplitude, gain }=0.5 \text {. }\end{array}$ & $\begin{array}{l}10 \text { shoulder flexion trials \& } \\
10 \text { shoulder extension trials, } \\
16.1 \mathrm{~cm} \text { amplitude gain }=0.5 \text {. }\end{array}$ \\
\hline $\begin{array}{l}\text { Block 3a \& 3b. Transfer } \\
\text { Test }\end{array}$ & $\begin{array}{l}15 \text { shoulder flexion trials \& } \\
15 \text { shoulder extension trials, } \\
16.1 \mathrm{~cm} \text { amplitudegain }=0.5 \text {. }\end{array}$ & $\begin{array}{l}15 \text { wrist flexion trials \& } 15 \\
\text { wrist extension trials, } 7.0 \\
\mathrm{~cm} \text { amplitude, gain }=0.5 \text {. }\end{array}$ \\
\hline
\end{tabular}


Table 2. Experiment Two Trial Descriptions

\begin{tabular}{|c|c|c|}
\hline Block Number & AA Group & HA Group \\
\hline Block 1a \& 1b. & $\begin{array}{l}15 \text { left to right trials \& } 15 \\
\text { right to left trials, head } \\
\text { movements, } 6.0 \mathrm{~cm} \\
\text { amplitude, gain=1.0. }\end{array}$ & $\begin{array}{l}15 \text { left to right trials \& } 15 \\
\text { right to left trials, arm } \\
\text { movements, } 6.0 \mathrm{~cm} \\
\text { amplitude, gain=1.0. }\end{array}$ \\
\hline Block 2a \& $2 b$ & $\begin{array}{l}15 \text { left to right trials \& } 15 \\
\text { right to left trials, arm } \\
\text { movements, } 6.0 \mathrm{~cm} \\
\text { amplitude, gain=1.0. }\end{array}$ & $\begin{array}{l}15 \text { left to right trials \& } 15 \\
\text { right to left trials, head } \\
\text { movements, } 6.0 \mathrm{~cm} \\
\text { amplitude, gain=1.0. }\end{array}$ \\
\hline A1a \& A1b: & $\begin{array}{l}20 \text { left to right trials \& } 20 \\
\text { right to left trials, } 8.4 \mathrm{~cm} \\
\text { amplitude, gain=0.5. }\end{array}$ & $\begin{array}{l}20 \text { left to right trials \& } 20 \\
\text { right to left trials, } 8.4 \mathrm{~cm} \\
\text { amplitude, gain=0.5. }\end{array}$ \\
\hline $\mathrm{A} 2 \mathrm{a} \& \mathrm{~A} 2 \mathrm{~b}:$ & $\begin{array}{l}15 \text { left to right trials \& } 15 \\
\text { right to left trials, } 6.6 \mathrm{~cm} \\
\text { amplitude, gain=0.5. }\end{array}$ & $\begin{array}{l}15 \text { left to right trials \& } 15 \\
\text { right to left trials, } 6.6 \mathrm{~cm} \\
\text { amplitude gain }=0.5 .\end{array}$ \\
\hline $\mathrm{A} 3 \mathrm{a} \& \mathrm{~A} 3 \mathrm{~b}:$ & $\begin{array}{l}10 \text { left to right trials \& } 10 \\
\text { right to left trials, } 4.8 \mathrm{~cm} \\
\text { amplitude, gain=0.5. }\end{array}$ & $\begin{array}{l}10 \text { left to right trials \& } 10 \\
\text { right to left trials, } 4.8 \mathrm{~cm} \\
\text { amplitude, gain=0.5. }\end{array}$ \\
\hline $\begin{array}{l}\text { Block 4a \& 4b. Transfer } \\
\text { Test }\end{array}$ & $\begin{array}{l}15 \text { left to right trials \& } 15 \\
\text { right to left trials, head } \\
\text { movements, } 7.2 \mathrm{~cm} \\
\text { amplitude, gain }=0.5 \text {. }\end{array}$ & $\begin{array}{l}15 \text { left to right trials \& } 15 \\
\text { right to left trials, arm } \\
\text { movements, } 7.2 \mathrm{~cm} \\
\text { amplitude, gain }=0.5 \text {. }\end{array}$ \\
\hline
\end{tabular}




\section{Figure Captions}

1. Experimental set-up. Subjects drew on a digitizing tablet with a cover over their arm to prevent them from viewing their hand moving. The start position, the target, and the pen-tip trajectory were displayed in real time to the subject on a computer monitor in front of them.

2. Sample trajectories. Individual trajectories from representative trials are plotted for early (upper row) and late (middle row) in adaptation, and upon transfer to the new effector (lower row). Early in adaptation, multiple adjustments are made to the trajectories. These are greatly diminished by the end of the adaptation block for both the wrist and shoulder pointing groups. Only small corrections are required upon transfer.

3. Primary submovement distance across the adaptation blocks. The block numbers are as defined in Table 1. Mean \pm standard error is plotted for the shoulder (group 1) and wrist (group 2) adaptation groups. There were significant linear and/or quadratic trends across trials for each adaptation block with the exception of block A3b (furthest right panel).

4. Transfer test primary submovement distance. The shoulder adaptation group has transferred to wrist pointing, and the wrist adaptation group has transferred to shoulder pointing. The left panel is block $3 \mathrm{a}$ (right-to-left movements) and the right panel is block $3 \mathrm{~b}$ (left-to-right movements, see Table 1). The $95 \%$ confidence intervals for performance on the first trial demonstrated that subjects covered substantially more than $50 \%$ of the target distance with the primary submovement. 
5. Sample trajectories. Individual trajectories from representative trials are plotted for early (upper row) and late (middle row) in adaptation and upon transfer to the new effector (lower row). Early in adaptation, multiple adjustments are made to the trajectories. These are greatly diminished by the end of the adaptation block for both the head and arm adaptation groups. The HA group exhibits segmented trajectories upon transfer, reflecting little transfer of adaptation from the head to the arm. The AA group presents trajectories similar to those late in adaptation, reflecting high transfer of adaptation from the arm to the head.

6. Primary submovement distance across the adaptation blocks for the head (HA) and arm (AA) adaptation groups. The blocks are as defined in Table 2. There were significant linear and quadratic trends across trials for the first two blocks, and a group main effect in block A2b (fourth panel).

7. Post test primary submovement distance. The left panel is block $4 \mathrm{a}$ (left-to-right movements) and the right panel is block $4 \mathrm{~b}$ (right-to-left movements, see Table 3). The $95 \%$ confidence interval for performance on the first trial was above $50 \%$ of the target distance for the AA group, reflecting transfer of adaptation from the arm to the head. The $95 \%$ confidence interval for the first trial included $50 \%$ of the target distance for the HA group, reflecting only little transfer of adaptation from the head to the arm. 


\section{References}

Abrams RA, Dobkins RS, Helfrich MK (1992) Adaptive modification of saccadic eye movements. J Exp Psychol Hum Percept Perform 18: 922-933

Berthoz A, Melvill Jones G (eds.) (1985) Adaptive Mechanisms in Gaze Control. Elsevier Biomed Press, Amsterdam

Bloomberg JJ, Merkle LA, Barry SR, Huebner WP, Cohen HS, Mueller SA, Fordice J (2000) Effects of adaptation of vestibulo-ocular reflex function on manual target localization. J Vestib Res 10: 75-86

Bloomberg JJ, Peters BT, Smith SL, Huebner WP, Reschke MF (1997) Locomotor head-trunk coordination strategies following space flight. J Vestib Res 7: 161-177

Caminiti R, Johnson PB, Galli C, Ferraina S, Burnod Y (1991) Making arm movements within different parts of space: The premotor and motor cortical representation of a coordinate system for reaching to visual targets. J Neurosci 11: 1182-1197

Carson RG, Goodman D, Elliott D (1992) Asymmetries in the discrete and pseudocontinuous regulation of visually guided reaching. Brain and Cognition 18: $169-191$

Cunningham HA Welch RB (1994) Multiple concurrent visual-motor mappings: Implications for models of adaptation. JExpPysch: HPP 20: 987-999

Darling WG, Butler AJ, Williams TE (1996) Visual perceptions of head-fixed and trunkfixed anterior/posterior axes. Exp Brain Res 112: 127-134

Darling WG Miller GF (1995) Perception of arm orientation in three-dimensional space. Exp Brain Res 102: 495-502

Darling WG Hondzinski JM (1997) Visual perceptions of vertical and intrinsic 
longitudinal axes. Exp Brain Res 116: 485-492

de Graaf JB, Pelisson D, Prablanc C, Goffart L (1995). Modifications in end positions of arm movements following short term saccadic adaptation. Neuroreport 6: 1733-1736

Dounskaia NV, Swinnen SP, Walter CB, Spaepen AJ, Verschueren SM (1998)

Hierarchical control of different elbow-wrist coordination patterns. Exp Brain Res 121: 239-254

Elliott D, Carson RG, Goodman D, Chua R (1991) Discrete vs. continuous visual control of manual aiming. Human Move Sci 10: 393-418

Flanders M, Helms Tillery SI, Soechting JF (1992) Early stages in a sensorimotọr transformation. Behav Brain Sci 15: 309-362

Georgopoulos AP, Kalaska JF, Caminiti R, Massey JT (1982) On the relations between the direction of two-dimensional arm movements and cell discharge in primate motor cortex. J Neurosci 2: 1527-1537

Gnadt JW, Bracewell RM, Andersen RA (1991) Sensorimotor transformation during eye movements to remembered visual targets. Vision Res 31: 693-715

Graziano MSA, Hu XT, Gross CG (1997) Visuospatial properties of ventral premotor cortex. J Neurophysiol 77: 2268-2292

Henriques DYP, Klier EM, Smith MA, Lowy D, Crawford JD (1998) Gaze-centered remapping of remembered visual space in an open-loop pointing task. J Neurosci 18: $1583-1594$

Huynh H, Feldt LS (1970) Conditions under which the mean square ratios in repeated measurements designs have exact F-distributions. J Am Stat Assoc 65: 1582-1589 Inoue K, Kawashima R, Satoh K, Kinomura S, Goto R, Sugiura M, Ito M, Fukuda H 
(1997) Activity in the parietal area during visuomotor learning with optical rotation. NeuroReport 8: 3979-3983

Ito M (1982) Cerebellar control of the vestibulo-ocular reflex around the floccular hypothesis. Annu Rev Neurosci 5: 275-296

Jeannerod M (1988) The Neural and Behavioural Organization of Goal-Directed Movements. Oxford: Clarendon Press

Kagerer FA, Contreras-Vidal J, Stelmach GE (1997) Adaptation to gradual as compared with sudden visuo-motor distortions. Exp Brain Res 115: 557-561

Lacquaniti F, Guigon E, Bianchi L, Ferraina S, Caminiti R (1995) Representing spatial information for limb movement: Role of Area 5 in the monkey. Cereb Cortex 5: 391-409

Lacquaniti F, Ferrigno G, Pedotti A, Soechting JF, Terzuolo C (1987) Changes in spatial scale in drawing and handwriting: Kinematic contributions by proximal and distal joints. J Neurosci 7: 819-828

Lacquaniti F, Soechting JF (1982) Coordination of arm and wrist motion during a reaching task. J Neurosci 2: 399-408

Mergner T, Huber W, Becker W (1997) Vestibular-neck interaction and transformation of sensory coordinates. J Vestib Res 4: 347-367

Meyer DE, Abrams RA, Kornblum S, Wright CE, Smith JEK (1988) Optimality in human motor performance: Ideal control of rapid aimed movements. Psychol Rev 95: $340-370$

Miles FA, Lisberger SG (1981) Plasticity in the vestibulo-ocular reflex. A new hypothesis: . Annu Rev Neurosci 4: 273-299 
Morasso P (1981) Spatial control of arm movements. Exp Brain Res 42: 223-227

Petrides M, Iversen SD (1979) Restricted posterior parietal lesions in the rhesus monkey and performance on visuospatial tasks. Brain Res 161: 63-77

Pine ZM, Krakauer JW, Gordon J, Ghez C (1996) Learning of scaling factors and reference axes for reaching movements. NeuroReport 7: 2357-2361

Pozzo T, Berthoz A, Lefort L (1990) Head stabilization during various locomotor tasks in humans. I. Normal Subjects. Exp Brain Res 82: 97-106

Pozzo T, Berthoz A, Lefort L, Vitte E (1991) Head stabilization during various locomotor tasks in humans. II. Patients with bilateral peripheral vestibular deficits. Exp Brain Res 85: 208-217

Prablanc C, Jeannerod M, Tzavaras A (1975a) Independent and interdependent processes in prism adaptation. In Vital-Durand F, Jeannerod M (Eds.), Aspects of Neural Plasticity. Paris: INSERM: 139-152

Prablanc C, Tzavaras A, Jeannerod M (1975b) Adaptation of the two arms to opposite prism displacements. Q J Exp Psychol 27: 667-671

Redding GM, Rader SD, Lucas DR (1992) Cognitive load and prism adaptation. J Mot Behav 24(3): 238-246

Redding GM, Wallace B (1988) Components of prism adaptation in terminal and concurrent exposure: Organization of the eye-hand coordination loop. Percept Psychophys 44: 59-68

Sakata H, Kusunoki M (1992) Organization of space perception: neural representation of three-dimensional space in the posterior parietal cortex. Curr Opin Neurobiol 2: 170174 
Seidler RD, Stelmach GE (in press) Trunk-assisted prehension: Specification of body segments with imposed temporal constraints. J Motor Behav

Seidler-Dobrin RD, Stelmach GE (1998) Persistence in visual feedback control by the elderly. Exp Brain Res 119: 467-474

Soechting JF, Flanders M (1991) Arm movements in three-dimensional space: computation, theory, and observation. Exerc Sport Sci Rev 19: 389-418

Teasdale N, Bard C, Fleury M, Young D, Proteau L (1993) Determining movement onsets from temporal series. J Mot Behav 25: 97-106

Wallace B (1978) Visuomotor coordination and intermanual transfer for a proprioceptive reaching task. J Mot Behav 10: 139-147

Walker N, Philbin DA, Fisk AD (1997) Age-related differences in movement control: Adjusting submovement structure to optimize performance. J Gerontol B Psychol Sci Soc Sci 52B: P40-P52

Winter DA (1990) Biomechanics and motor control of human movement (2nd ed.). John Wiley \& Sons 


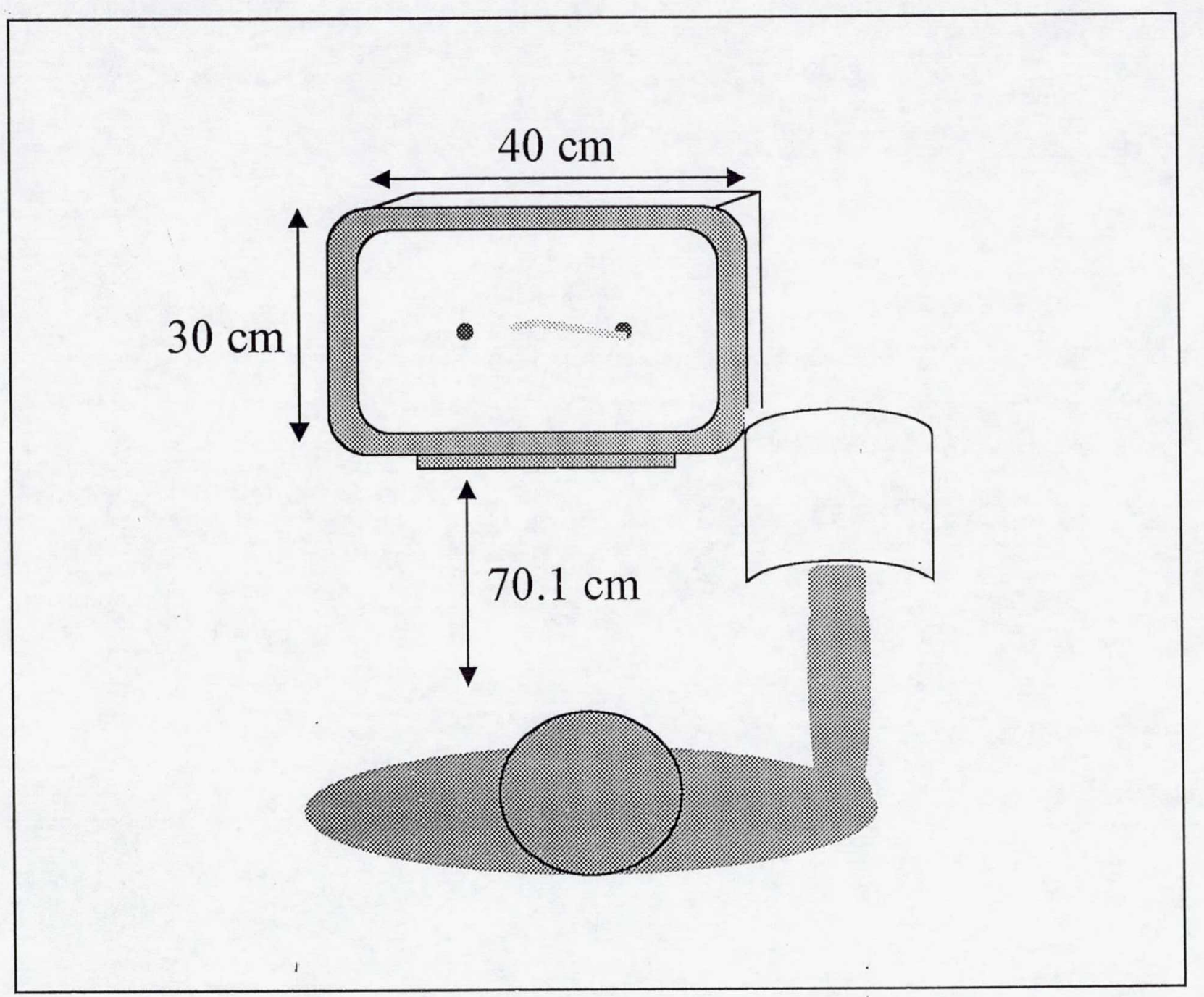

Fig. 1 
Group 1 (SA)
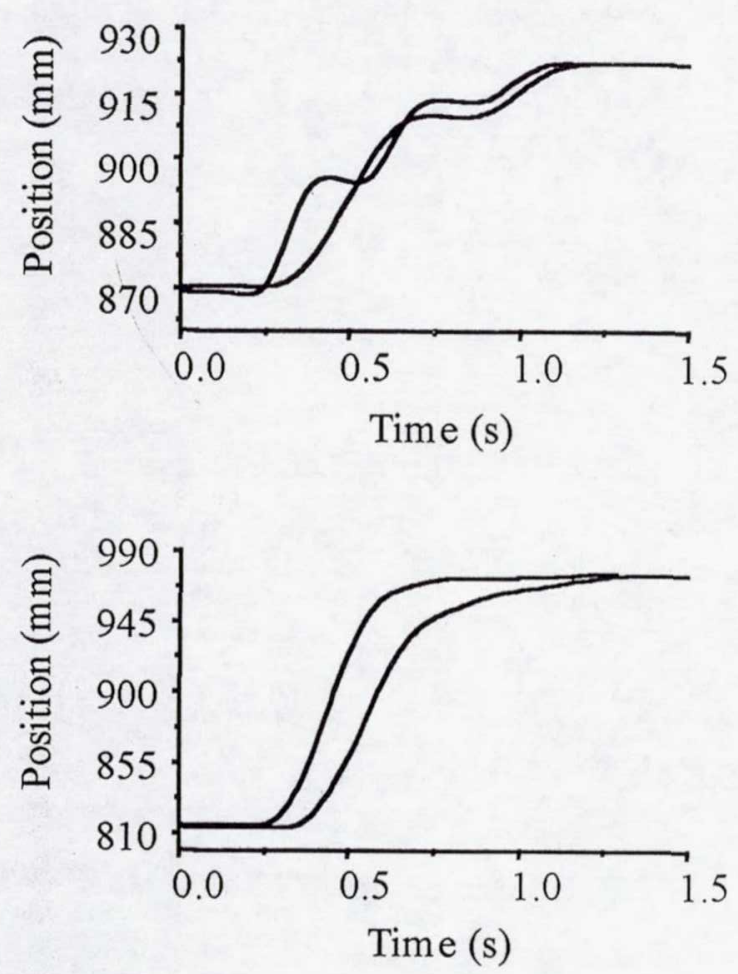

late in

adaptation

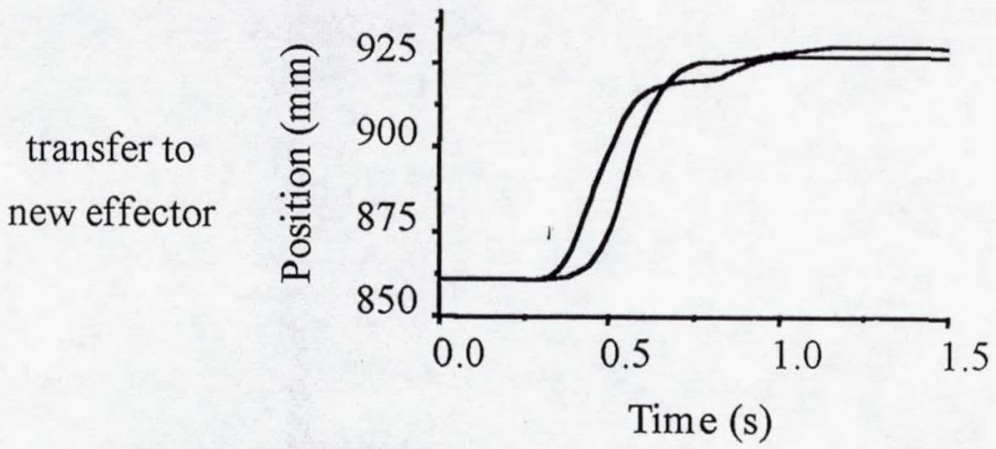

Group 2 (WA)
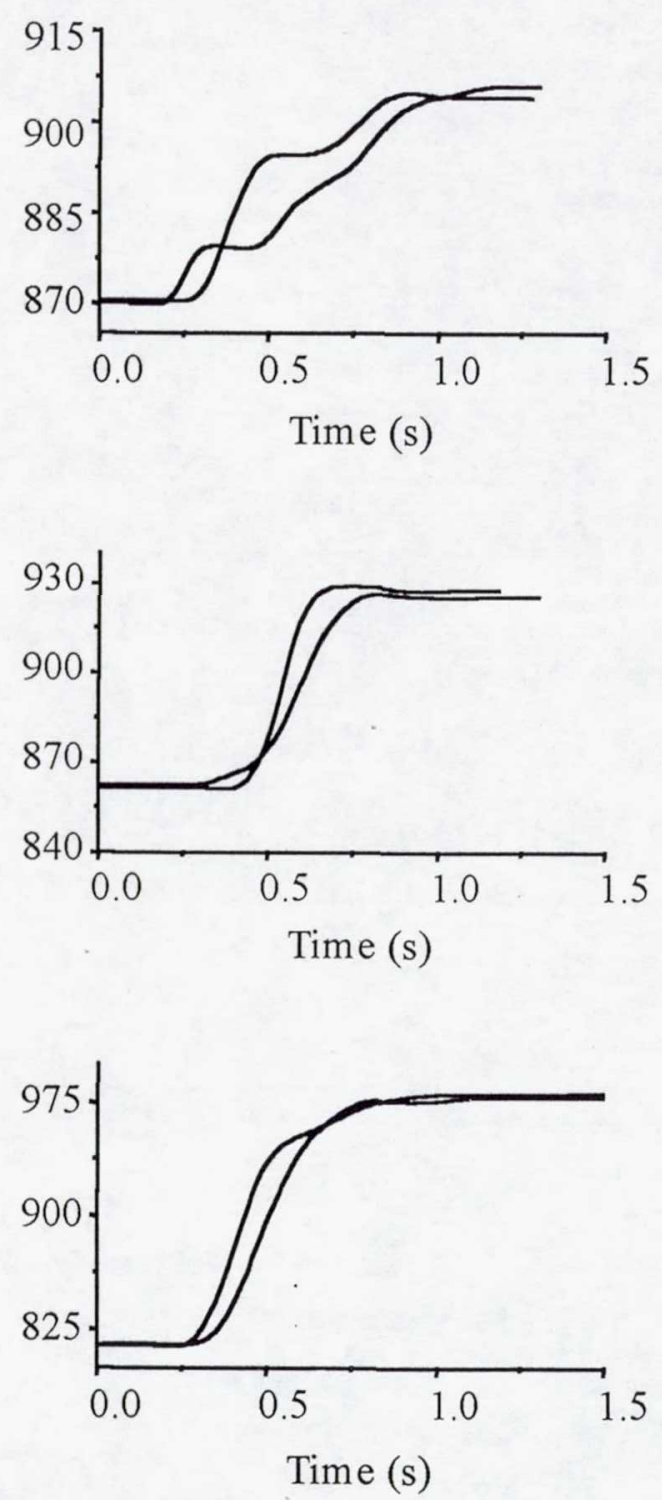

$$
\text { Fig. } 2
$$




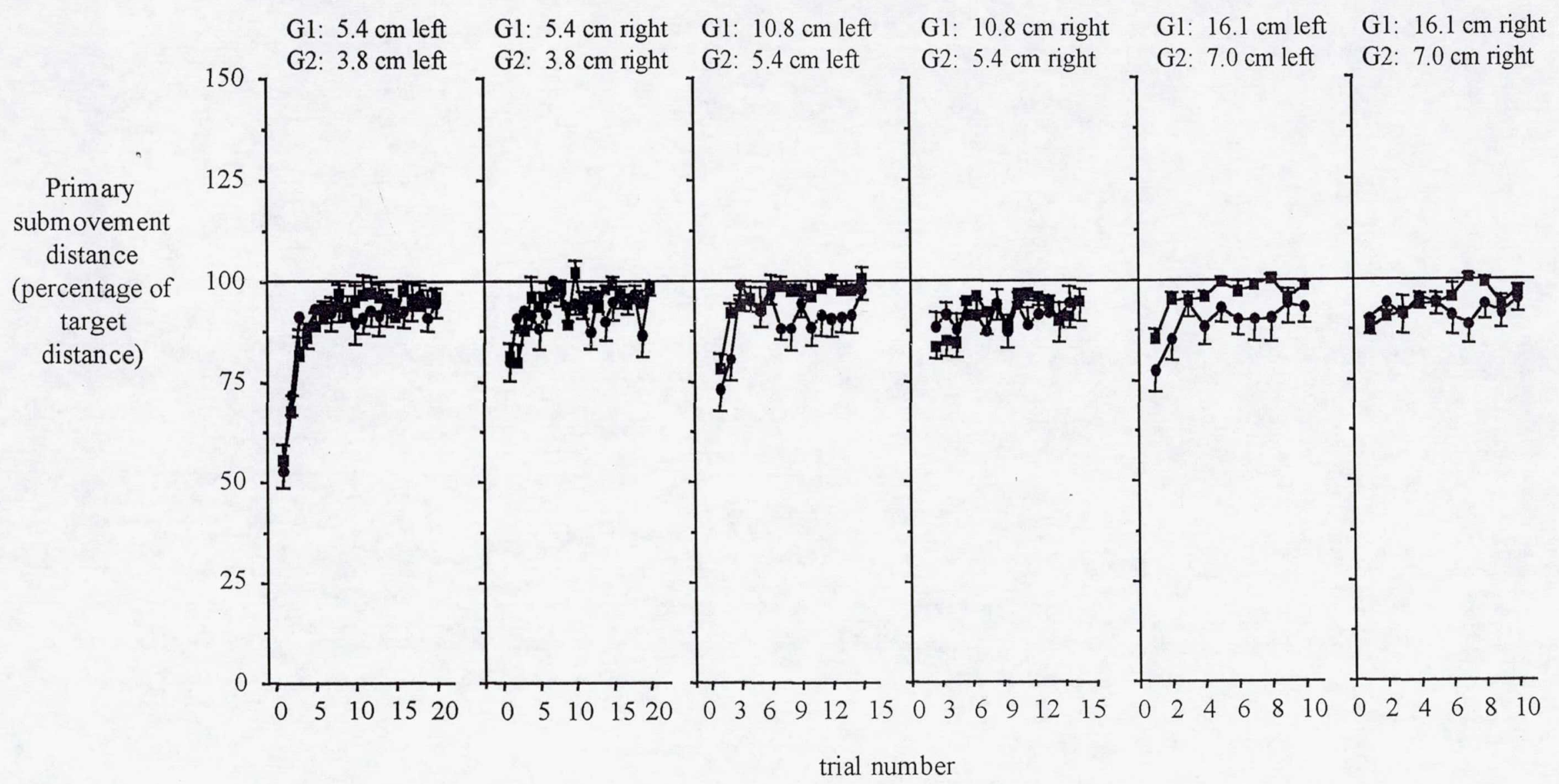

- Group 1 (Shoulder Adaptation)

-— Group 2 (Wrist Adaptation) 


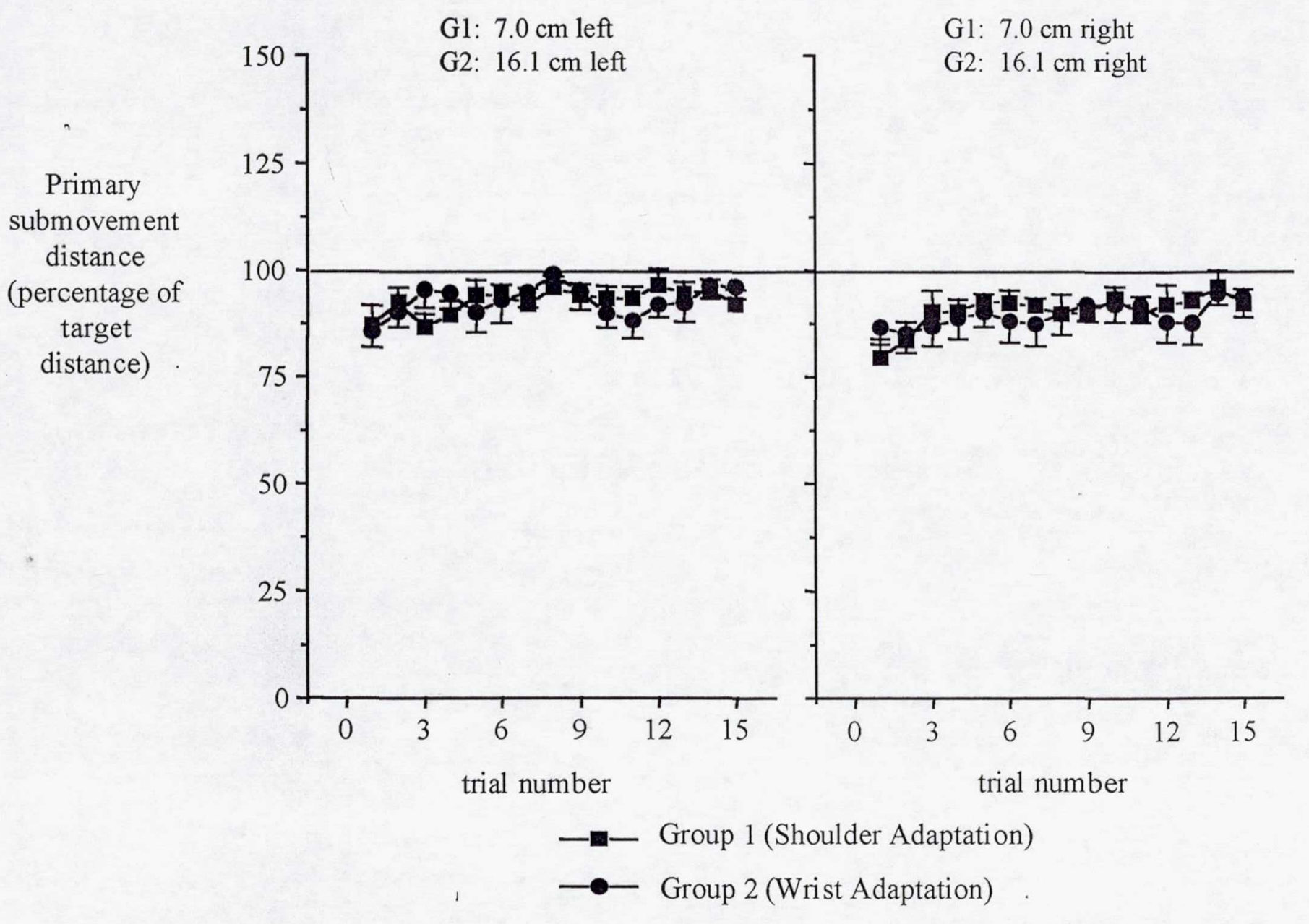

Fig. 4 

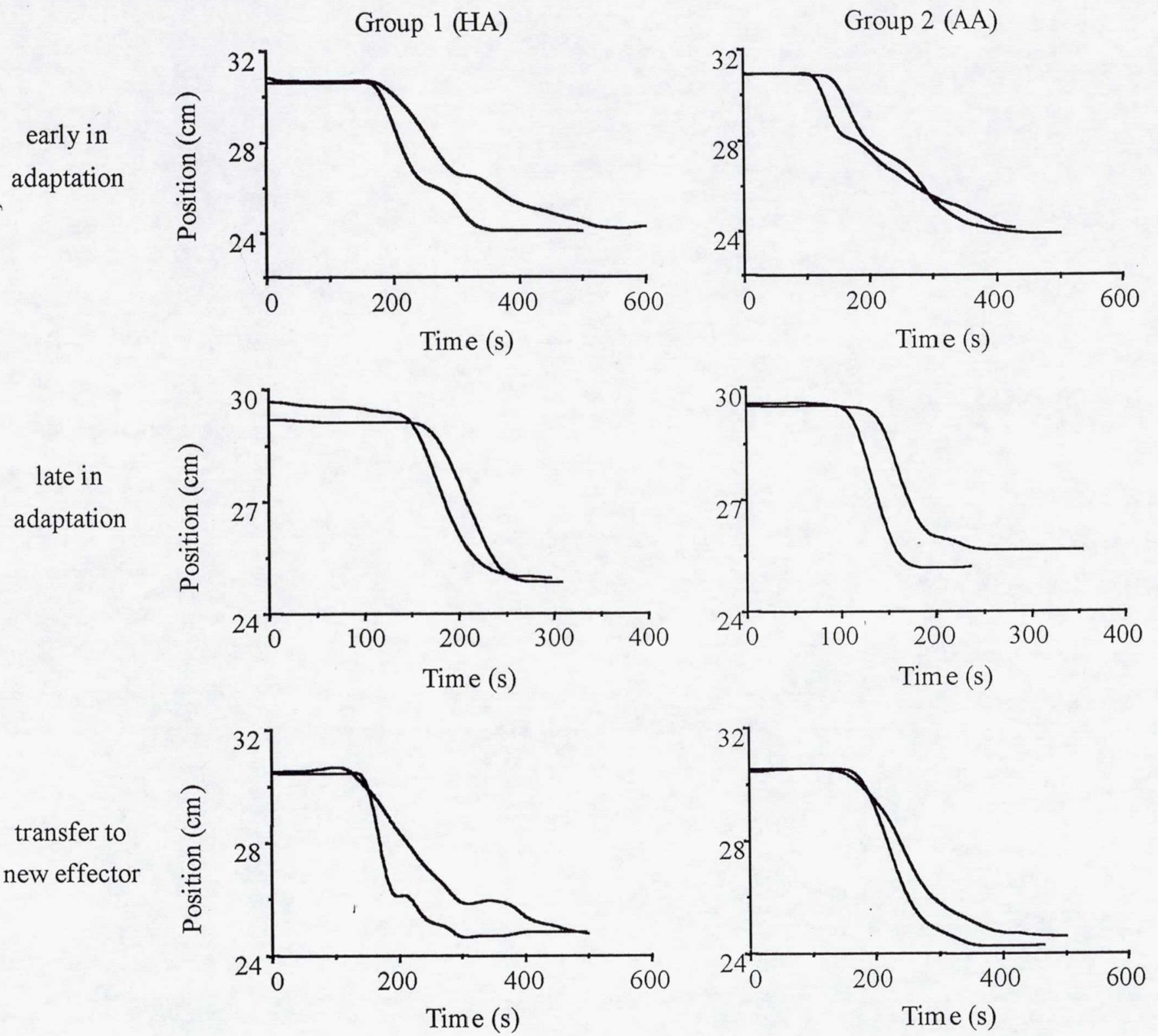


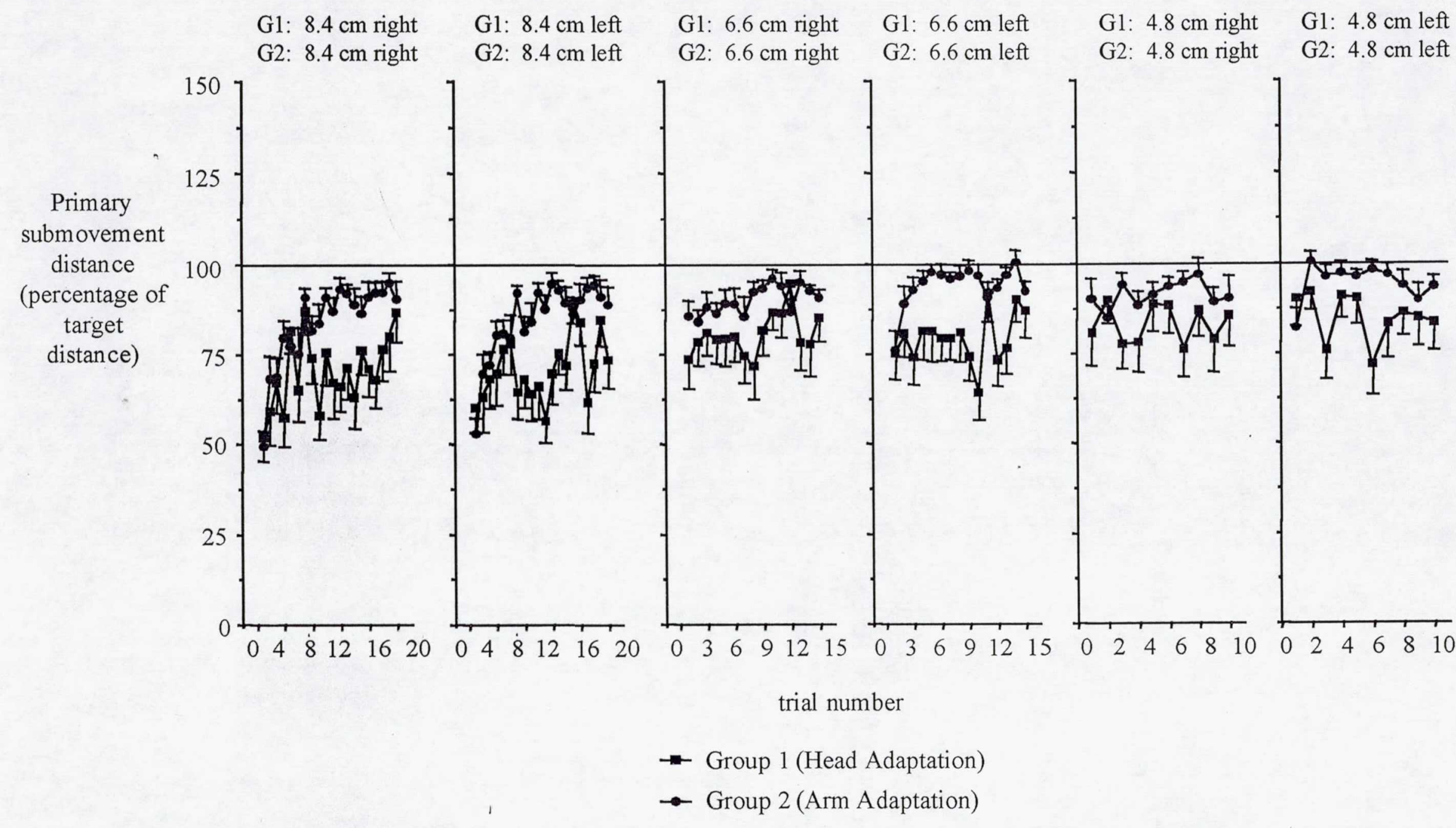

Fig. C 


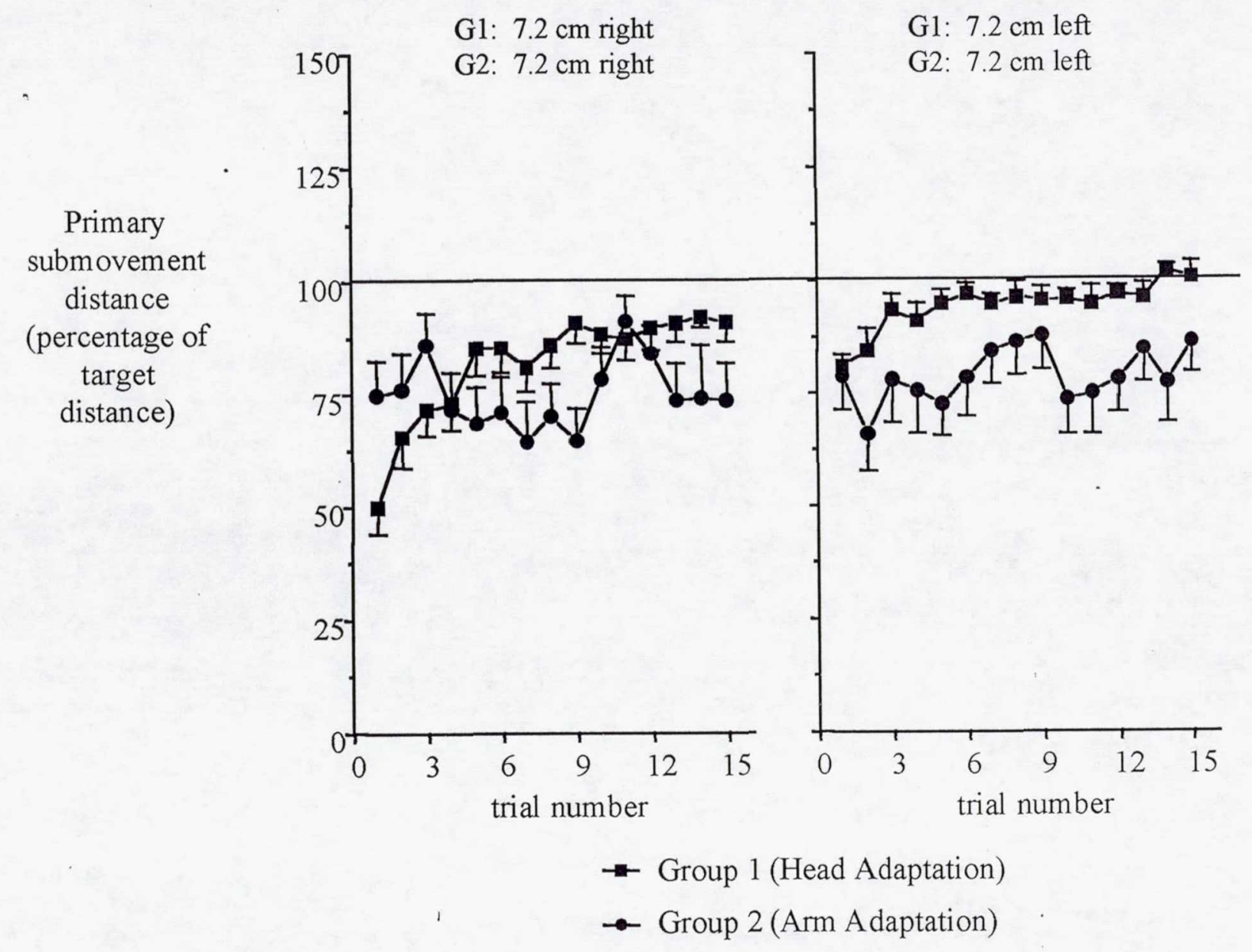

Fig. 7 